Pecvnia, 4 (2007), pp. 81-107

\title{
Una Experiencia de Aprendizaje en el Nuevo Contexto del EEES: el Caso del Webinar de SICODINET sobre Modelización con Hoja de Cálculo para la Información de Gestión Empresarial
}

\author{
Begoña González Pérez \\ b.gonzalez@unileon.es \\ Enrique López González \\ enrique.lopez@unileon.es \\ Cristina Mendaña Cuervo \\ cristina.mendana@unileon.es \\ Universidad de León \\ Departamento de Dirección y Economía de la Empresa \\ Facultad de Ciencias Económicas y Empresariales \\ Campus de Vegazana $s / n$ \\ 24071 León (España)
}

Los cambios en la educación universitaria que propicia el Espacio Europeo de Educación Superior implican nuevas adaptaciones en el proceso de enseñanza-aprendizaje ya que el objetivo primordial pasa a ser "aprender a aprender". En este contexto, se necesitan nuevos medios que faciliten el desarrollo de tales cambios, que permitan realizar actividades adicionales a la enseñanza presencial. Así, las nuevas Tecnologías de la Información y la Comunicación constituyen herramientas que posibilitan tales transformaciones. En este sentido, los Espacios Virtuales
The European Higher Education Area has caused changes in higher education, what involves new adaptations in the teachinglearning process because now the main aim is "learn to learn". So, new means are needed for making easy these changes and designing other activities, besides face to face classes. New Information and Communication Technologies (ICTs) are tools which make easy these transformations. In this sense, Virtual Learning Spaces (VLS) make possible 
Una Experiencia de Aprendizaje en el Nuevo Contexto del EEES: el Caso del Webinar de SICODINET sobre Modelización con Hoja de Cálculo para la Información de Gestión Empresarial

\begin{abstract}
de Aprendizaje (EVA) posibilitan la creación de comunidades sociales donde se intercambian conocimientos. Este trabajo se centra en el EVA SICODINET que se ha diseñado en torno a las asignaturas del perfil de Contabilidad de Costes y de Gestión de la Universidad de León. Concretamente se profundiza en la experiencia del seminario en línea (Webinar) sobre hoja electrónica de cálculo celebrado durante tres años, sus características, la metodología seguida, los resultados obtenidos y las lecciones aprendidas con esta iniciativa.
\end{abstract}

Palabras clave: Contabilidad de Costes, Espacio Europeo de Educación Superior (EEES), Espacio Virtual de Aprendizaje (EVA), Seminario en Línea, Hoja de Cálculo, Modelos de Gestión. the creation of social communities where knowledge is exchanged. This paper analyzes the VLS SICODINET which has been designed around Cost and Management Accounting subjects in the University of León. Specifically the Web-based seminar (Webinar) about spreadsheet is studied in depth: it has been held for three years and its characteristics, the followed methodology, its results and the lessons learnt from this initiative are explained.

Key words: Cost Accounting, European Higher Education Area, Virtual Learning Space (VLS), Webinar, Spreadsheet, Management Models.

\section{INTRODUCCIÓN}

Las nuevas Tecnologías de la Información la Comunicación (TICs) se han introducido en la enseñanza superior provocando modificaciones en el tradicional proceso de enseñanza-aprendizaje; el aula donde coinciden alumnos y profesor ha dejado de ser el único contexto en el que produce transmisión de conocimientos e interacción entre ambas figuras.

El uso de las TICs cada día se generaliza más en el ámbito universitario, siendo numerosas las experiencias en las que únicamente Internet se utilizado como soporte para el aprendizaje (electronic-learning) o como apoyo a la enseñanza presencial (blended-learning).

Además, las TICs constituyen herramientas de suma importancia dentro del Espacio Europeo de Educación Superior (EEES) ya que su manejo se sitúa como uno de los puntos fundamentales para la convergencia educativa europea. Asimismo, las TICs se conforman como instrumentos de gran utilidad para la aplicación y el desarrollo del eje central del EEES: el alumno se convierte en un personaje activo en su propio proceso de aprendizaje, mientras que el profesor es sólo un guía, un facilitador que conduce al estudiante para que descubra el conocimiento. El objetivo no se restringe únicamente a transmitir conocimientos, sino que también propicia la adquisición de habilidades y capacidades por parte de los alumnos, como el trabajo en equipo, la comunicación, etc. 
En este contexto, la clase presencial y la lección magistral dejan de ser el único medio para valorar la carga de las asignaturas y cada vez más las horas de estudio y trabajo de los alumnos se constituyen en referentes de tal valoración. Por ello, actividades como seminarios, tutorías personalizadas o trabajos en equipo también se consideran con el objetivo de computar todo el esfuerzo realizado por el alumno para superar una asignatura. En consecuencia, se hacen necesarios nuevos escenarios que constituyan un apoyo al aprendizaje de los alumnos, en los que puedan desarrollar sus habilidades.

Los "Espacios Virtuales de Aprendizaje" (EVA) o "Portales de Conocimiento" son las expresiones más avanzadas de la evolución de las TICs en el contexto educativo ya que permiten la creación de comunidades sociales para la comunicación y el trabajo colaborativo de alumnos y profesores. En este ámbito se inserta la propuesta del EVA denominado "Sistemas de Información para el Control y la Dirección en Entornos Telemáticos (SICODINET)", cuyo objetivo principal es aplicar las tecnologías emergentes de e-learning en la enseñanza de las asignaturas del perfil docente de "Contabilidad de Costes y de Gestión" en la Universidad de León. Precisamente, en estas asignaturas, la hoja electrónica de cálculo tiene vital importancia por tratarse de un instrumento que permite realizar simulaciones y facilita la toma de decisiones. Por ello, en SICODINET (http://sicodinet.unileon.es) se ha implementado el Webinar: "La hoja electrónica de cálculo como herramienta de apoyo a la elaboración de información de gestión", que constituye el eje central y que orienta el presente trabajo, cuyo propósito es analizar esta experiencia de innovación docente universitaria y extraer algunas conclusiones para generalizar dentro del EEES.

\section{EL ESPACIO EUROPEO DE EDUCACIÓN SUPERIOR}

El Espacio Europeo de Educación Superior (EEES) es un proyecto impulsado por la Unión Europea para armonizar los sistemas universitarios europeos, de manera que la estructura de titulaciones, los estudios de formación continua, la valoración de la carga lectiva y el sistema de calificaciones sean homogéneos y fácilmente entendibles en todos los estados miembros de la UE. El objetivo que se persigue es dotar a Europa de un sistema universitario homogéneo, compatible y flexible, que permita a los estudiantes y titulados universitarios europeos una 
mayor movilidad, al igual que establecer unos niveles de transparencia y calidad, mediante sistemas de evaluación, que lo hagan atractivo y competitivo a nivel internacional.

El EEES se inició con la Declaración de la Sorbona (mayo de 1998), con sólo cuatro países signatarios, y los últimos pasos dados en el proceso fueron la Conferencia de Berlín (septiembre de 2003), donde más de cuarenta representantes de países, instituciones y asociaciones firmaron el documento final, la Conferencia de Bergen (mayo de 2005) y la Reunión de Londres (mayo de 2007).

El año 2010 es la fecha clave para la implantación generalizada de todos los criterios y directrices que conforman las bases del EEES. La Declaración de Bolonia (1999) constituye el marco de referencia del proceso de convergencia educativa, siendo sus principios más destacados los siguientes:

- La adopción de un sistema comparable de titulaciones.

- El desarrollo de un sistema de estudios basado en dos niveles: grado y postgrado.

- El sistema de créditos europeo (European Credits Transfer System -ECTS-).

- La eliminación de las barreras educativas existentes entre países europeos, promoviendo la movilidad de estudiantes, profesores e investigadores.

- La promoción del aprendizaje continuado (lifelong learning).

Entre los aspectos citados, quizás sea la implantación de un sistema de créditos común uno de los más destacados para desarrollar el espacio común de educación superior. Esta medida es ineludible para un reconocimiento de estudios y títulos que permita la movilidad de estudiantes y profesores. Asimismo, este sistema común facilita la comparabilidad y garantiza la calidad de los estudios cursados dentro del EEES.

Un ECTS es la unidad de medida por la que se rige el nuevo sistema académico en la Unión Europea. En España el Real Decreto 1125/2003 de 5 de septiembre (BOE de 18 de septiembre) define el ECTS como:

la unidad de medida del haber académico... en la que se integran las enseñanzas teóricas y prácticas, así como otras actividades académicas dirigidas, con inclusión de 
las horas de estudio y de trabajo que el estudiante debe realizar para alcanzar los objetivos formativos propios de cada una de las materias del correspondiente plan de estudios.

Hasta la actualidad y en el caso concreto de España -similar a muchos sistemas educativos- los actuales créditos miden únicamente las horas lectivas (artículo 2.7. del Real Decreto 1497/1987 de 27 de noviembre): cada crédito está establecido en 10 horas lectivas teóricas, prácticas o equivalencias. Frente a esta concepción, el ECTS mide tanto las horas de clase teóricas y prácticas como el esfuerzo dedicado al estudio, la preparación y la realización de exámenes por parte del estudiante para superar una materia. En concreto, se dispone que cada ECTS se compondrá de 25 ó 30 horas de trabajo (Real Decreto 1125/2003 de 5 de septiembre), estableciendo que los créditos para la obtención de un título universitario de grado se sitúan en 180 ó 240 , según la duración de estas titulaciones sea de 3 ó 4 años, respectivamente, si bien el actual gobierno ha propuesto la segunda opción.

Por tanto, el nuevo sistema de educación superior sitúa al estudiante como centro del proceso de enseñanza-aprendizaje, al valorar no sólo las horas presenciales de docencia, sino también el esfuerzo total requerido para superar cada materia. En este sentido, en la programación de las asignaturas se consideran estas circunstancias y el profesorado debe incluir, además de las horas de docencia, el tiempo dedicado a organizar, orientar y supervisar el trabajo de los alumnos. De hecho, las horas de docencia teórico-prácticas no podrán superar nunca el $50 \%$ de los créditos asignados a cada materia.

En resumen, la nueva unidad de medida, ECTS, refleja un nuevo modelo educativo basado en el trabajo del alumno y en la adquisición de competencias y destrezas y no en las horas de clase, o expresado de otro modo, centrado en el aprendizaje de los estudiantes y el esfuerzo que requiere el mismo. Por este motivo se precisa un cambio en la actitud del propio estudiante y del profesor, siendo el objetivo principal del proceso educativo que el alumno "aprenda a aprender", buscando un aprendizaje continuado. El conocimiento de las diferentes materias debe tener como propósito que los fundamentos básicos, habilidades, aptitudes y actitudes (competencias) necesarias se desarrollen dentro de un ámbito estimulante. El proceso educativo debe estar influido tanto por la adquisición de competencias transversales (instrumentales, personales y sistémicas) como 
Una Experiencia de Aprendizaje en el Nuevo Contexto del EEES: el Caso del Webinar de SICODINET sobre Modelización con Hoja de Cálculo para la Información de Gestión Empresarial

por el conocimiento de los contenidos propios de cada asignatura (competencias específicas).

Como se ha mencionado anteriormente, con la introducción del ECTS se pasará a valorar las horas de trabajo del estudiante en lugar del tiempo de docencia del profesor. El profesor tiene que desempeñar nuevas tareas reconocidas, además de la impartición de clases, como las tutorías, la atención personalizada, la organización, desarrollo y evaluación de tareas prácticas, etc. (dedicadas todas ellas a organizar, orientar y supervisar el trabajo de los estudiantes). Todo ello supone un reconocimiento del tiempo total que el profesor debe emplear en la docencia de modo general, y no únicamente a las clases presenciales.

La enseñanza universitaria necesita ahora más dedicación y atención ya que los profesores deberán desarrollar nuevas actividades, como trabajos prácticos, seminarios, etc. para adaptarse al nuevo entorno educativo. El desarrollo de las mismas se verá facilitado con la utilización de las TICs porque, como es lógico, la utilización de herramientas como e-mail, foros o chats, entre otras, agilizará el proceso de tutorías entre profesores y alumnos, además de facilitar la labor del profesor a la hora de supervisar y organizar el aprendizaje de los alumnos. Igualmente herramientas como pizarras digitales, portales y aulas virtuales mejoran la labor de enseñanza del profesor al poner a su disposición medios que le permiten organizar y suministrar información a los alumnos, independientemente del lugar en que se encuentren físicamente y del momento en que decidan dedicar su tiempo al aprendizaje. Las TICs favorecen la interacción entre profesores y alumnos construyendo un aprendizaje cooperativo.

Asimismo, los ordenadores, Internet y el resto de TICs facilitan también la formación continua del profesorado universitario; los profesores tienen acceso a más información y pueden gestionarla mejor. También se posibilita la cooperación entre docentes, propiciándose el intercambio de información y de ideas y la colaboración en proyectos comunes de docencia y de investigación.

En este contexto, las TICs constituyen herramientas de gran utilidad para realizar la transformación requerida en el proceso de enseñanza-aprendizaje universitario. En consecuencia, se necesitan adaptaciones tecnológicas (además de las adaptaciones curriculares obvias) que incorporen la utilización de las nuevas tecnologías en la enseñanza superior como mecanismo para facilitar el proceso de aprendizaje interdependiente del alumno, así como la valoración de su trabajo 
personal y de sus actividades no presenciales. Por ello, los docentes deben aprender a enseñar con nuevas metodologías, adaptando su proceso de enseñanza a los nuevos requerimientos que se propugnan desde el EEES.

\section{EL PROYECTO SICODINET}

El Espacio Virtual de Aprendizaje (EVA) denominado SICODINET, cuya página de acceso puede observarse en la Figura 1, es una plataforma edumática que surge inicialmente ante la necesidad de realizar un proceso de adaptación de las tradicionales técnicas de enseñanza en el ámbito universitario a la nueva realidad que impone la sociedad de la información y al nuevo EEES.

Figura 1. Página de acceso al EVA

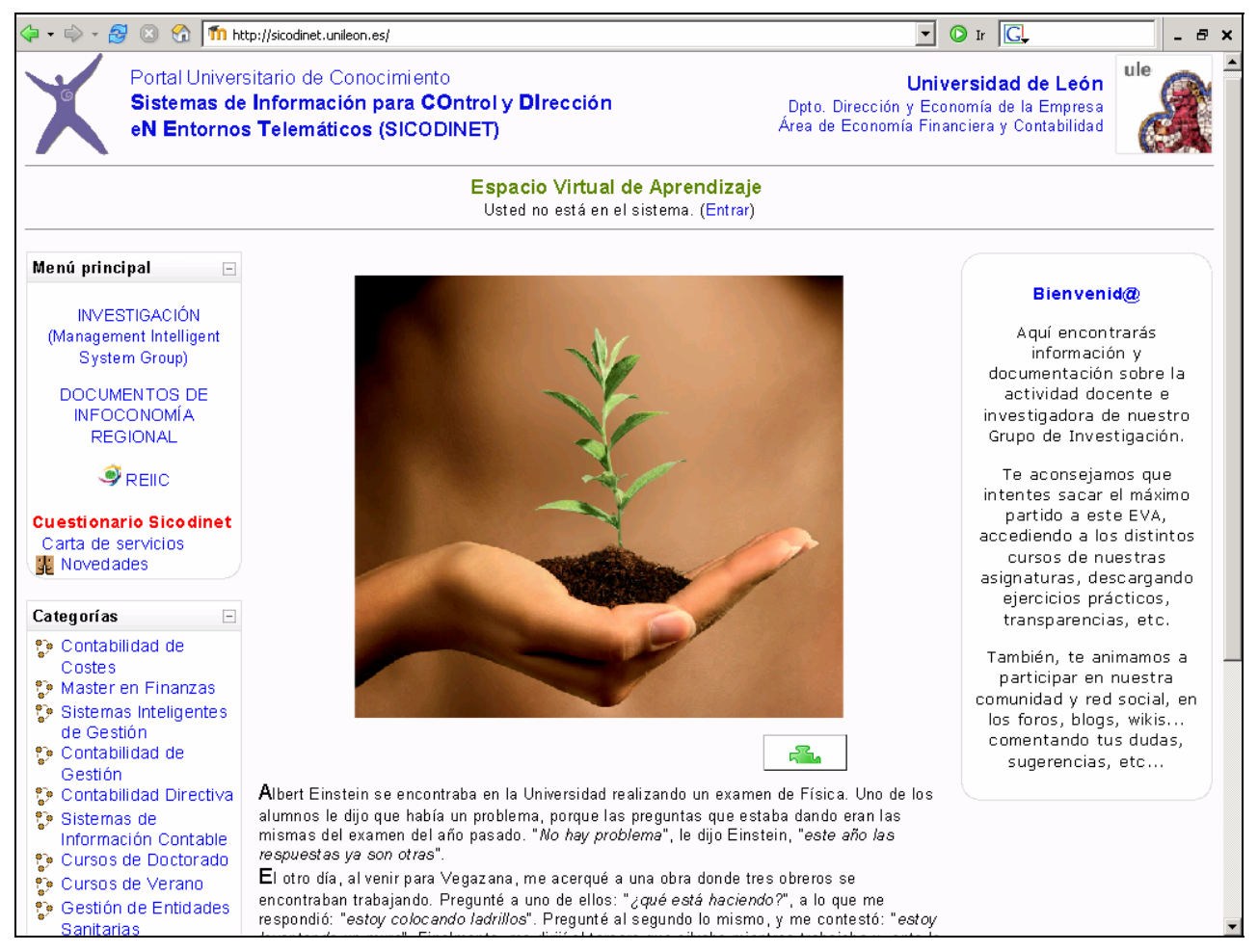

Como se ha expuesto, el diseño y desarrollo de SICODINET se ha fundamentado en los contenidos de las asignaturas que constituyen la oferta docente de perfil universitario anteriormente mencionado, en 
especial la asignatura de "Contabilidad de Costes", materia obligatoria del primer ciclo de la Licenciatura en Administración y Dirección de Empresas y troncal de la Diplomatura en Ciencias Empresariales. Esto ha implicado que la actividad sea accesible a un número elevado de alumnos y la necesidad de coordinar todas las asignaturas de dicho perfil, permitiendo a los alumnos un seguimiento completo de estas enseñanzas.

Asimismo, el EVA también se utiliza como complemento en la impartición de los distintos Cursos de Doctorado, en el desarrollo de talleres virtuales en Congresos o de los distintos seminarios en línea. En concreto, en la Figura 2 se puede observar los cursos que se han desarrollado en SICODINET en los últimos tres años.

Figura 2. Categorías de cursos en el EVA

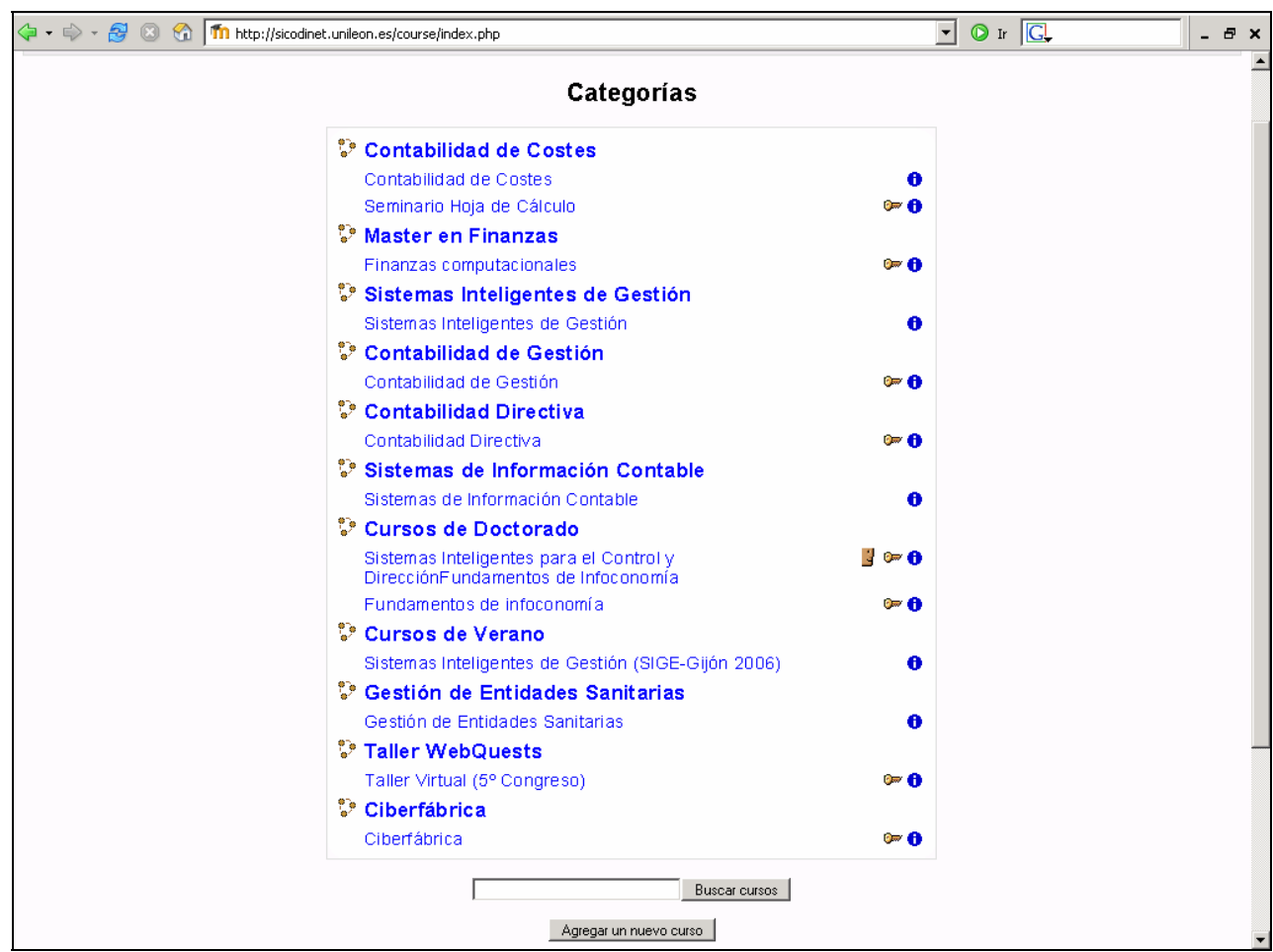

A tenor de lo anterior, con SICODINET se ha tratado de facilitar, por un lado, el apoyo a la enseñanza presencial y, por otro, los aspectos relativos a la gestión administrativa y docente de alumnos y asignaturas. Desde la primera perspectiva, SICODINET puede calificarse como una iniciativa de blended-learning, ya que en muchas ocasiones se 
ha empleado como apoyo en la enseñanza presencial, lo que ha permitido beneficiarse de las ventajas de las TICs, al tiempo que los profesores asumen su papel tradicional en la docencia presencial. La segunda perspectiva es muy valiosa porque ha implicado dotar al EVA del dinamismo suficiente para que no sea simplemente un conjunto de materiales en red, sino que fomente la participación de los alumnos, agilice el proceso tutorial, etc., y permita que alumnos y profesores gestionen procesos administrativos.

La dotación de estas cualidades a SICODINET ha permitido crear un particular modelo de "portal universitario de conocimiento" como fue reconocido con la concesión del primer premio de Innovación a la Enseñanza 2005 del Consejo Social de la Universidad de León (http://sicodinet.unileon.es/dir/docs/sicodinet-innovacioneducativa.pdf).

Respecto a la implementación técnica, SICODINET se ha desarrollado en la plataforma Moodle (http://moodle.org). Tras realizarse un análisis de las diferentes plataformas existentes y revisar diversos estudios comparativos al respecto ${ }^{1}$, se optó por seleccionar Moodle por tratarse de software libre, gratuito y por sus ventajas técnicas, operativas y legales. Cabe destacar que Moodle cuenta con todas las herramientas necesarias para la gestión y administración de cursos, la comunicación sincrónica y asincrónica y la gestión de materiales y aprendizaje, permitiendo incluso el seguimiento y la evaluación de los alumnos. De forma detallada, las características que argumentan la selección de la plataforma Moodle son las siguientes:

- Tipología y número de herramientas, funcionalidades y recursos que presenta, lo que permite desarrollar diferentes estilos docentes.

- Diseño modular, que facilita la elección de diferentes tipos de recursos en función de las características de los contenidos que se deseen llevar a cabo.

- Usabilidad, ya que si bien el número de herramientas es muy elevado y la sofisticación sobre todo pedagógica de muchas de ellas es alta, la posibilidad de elección hace que el manejo sea simple, tanto desde el punto de vista del profesor y/o administrador, como para el usuario final.

\footnotetext{
Estudio del Gabinete de Tele-Educación de la Universidad Politécnica de Madrid (http://www.gate.upm.es); Herramienta para el estudio y comparativa de Sistemas de Gestión de Cursos elaborada por EduTools (http://edutools.info/course/compare/); Estudio realizado en la Universidad de Málaga sobre plataformas de Teleformación (http://campusvirtual.uma.es/ieev/filos.htm).
} 
Dinamismo de la comunidad de desarrolladores y usuarios de Moodle. La participación activa que se ha llevado a cabo durante el periodo de desarrollo del proyecto en los diversos foros ha permitido constatar no sólo la evolución que esta plataforma ha tenido en este periodo de tiempo, sino el grado de participación tanto en la toma de decisiones como en la resolución de problemas que se plantean al resto de miembros de la comunidad.

- Facilidad de actualización. En el periodo de tiempo en el que se lleva desarrollando el proyecto SICODINET bajo el entorno Moodle, aproximadamente 3 años, se han producido varias actualizaciones a versiones más avanzadas y con nuevas herramientas y recursos, pudiendo comprobarse la facilidad de actualización de esta plataforma.

\section{COSTES}

\section{LA HOJA ELECTRÓNICA DE CÁLCULO EN CONTABILIDAD DE}

La utilización de las hojas electrónicas en la enseñanza en general, y en la Contabilidad en particular, ha sido reconocida desde hace mucho tiempo por las ventajas que presenta, entre las que cabe destacar las siguientes:

- Suministran aplicaciones preestablecidas con las que enseñar su uso y demostrar el potencial que presentan las mismas.

- Ayudan a los estudiantes en la comprensión de situaciones complejas de Contabilidad, ya que la resolución de problemas con el concurso de estos instrumentos proporciona soluciones que cumplen una serie de características positivas, como facilitar la comprensión, asegurar la eficiencia en los resultados obtenidos o permitir el análisis de las situaciones planteadas.

Sin embargo, en la utilización de las hojas electrónicas se debe tener presente los posibles inconvenientes que pueden plantear tanto desde el punto de vista del alumno como desde la perspectiva del docente, tales como:

- La utilización de estos instrumentos requiere, en primer lugar, el aprendizaje del manejo de los mismos y, en segundo lugar, la aplicación a la problemática concreta de la Contabilidad de Gestión, 
lo cual implica un doble esfuerzo que requiere asimismo un mayor espacio de tiempo.

- El peligro de reducir la repetición de problemas prácticos, que en ciertos casos resulta necesaria, para la comprensión de los mismos.

No obstante, y dejando a un lado los anteriores inconvenientes, los objetivos que se persiguen en Contabilidad de Gestión con la utilización de la hoja de cálculo son principalmente dos: por un lado, la construcción de modelos y ejercicios de simulación y, por otro lado, facilitar la toma de decisiones de gestión.

En lo que se refiere al primer objetivo, la modelizaciónsimulación realizada a través de hoja de cálculo supone las siguientes ventajas:

- El alumno es entrenado a través de un mecanismo de autoaprendizaje. Primero, define de la manera más estructurada posible el problema a resolver; a continuación, determina con cuidado las variables que tienen impacto en la búsqueda de soluciones; y, por último, reflexiona sobre la naturaleza de las relaciones que unen las variables.

- La rapidez y facilidad con que se obtienen las soluciones, posibilita la realización de múltiples pruebas y el fácil aprendizaje del mecanismo estímulo-respuesta propio del fenómeno en estudio que, a su vez, debe provocar una motivación creciente de estudio de situaciones más complejas y más próximas a la realidad.

- La adaptación mimética del estudiante a los métodos habituales de análisis, que se ha de encontrar en su futuro puesto de trabajo.

En lo referente a la toma de decisiones de gestión, una ventaja del uso de hoja electrónica de cálculo consiste en la facultad de investigar las consecuencias de la modificación de parámetros específicos dentro del modelo; de hecho, esta posibilidad de preguntarse "qué pasaría si" con frecuencia es descrita como la principal contribución de esta herramienta. De esta forma, una vez que se ha desarrollado un modelo en hoja de cálculo, el foco de atención puede variar sobre las decisiones acerca de qué variables hay que manejar, del porqué dichas variables deberían ser manejadas y de sobre cómo interpretar los resultados.

Por todo lo expuesto con anterioridad, se consideró necesario realizar un seminario en el que alumnos aprendiesen a utilizar la hoja electrónica de cálculo y a aplicar la misma en la realización de la 
modelación-simulación para la toma de decisiones de gestión. Esta iniciativa se realizó en un primer momento con carácter presencial, pero en los tres últimos cursos académicos se ha desarrollado mediante modalidad on-line, dando lugar al concepto de Seminario Virtual o Webinar.

5. UNA EXPERIENCIA DE E-LEARNING: LOS WEBINAR DE SICODINET SOBRE HOJA DE CÁLCULO

\subsection{Descripción del Webinar}

Durante tres cursos académicos consecutivos (2004/2005, $2005 / 2006$ y 2006/2007) se ha implementado en SICODINET el Webinar "La hoja electrónica de cálculo como herramienta de apoyo a la elaboración de información de gestión". Este seminario, de carácter voluntario y gratuito, tiene su origen en una serie de seminarios previos que se desarrollaron durante varios cursos académicos, dirigidos prioritariamente a los alumnos de la asignatura de Contabilidad de Costes e impartidos como actividad presencial complementaria. Los seminarios presenciales tenían carácter eminentemente práctico y se realizaban en su totalidad con la utilización de sistemas informáticos, lo que supuso que, curso a curso, ante el elevado número de alumnos que participaban y las dificultades de disposición física de puestos de trabajo en las salas de ordenadores de la Facultad de Ciencias Económicas y Empresariales, durante el curso académico 2004/2005 simultáneamente se desarrolló la modalidad virtual, y desde el curso académico 2005/2006 se lleva a cabo únicamente en esta última.

El Webinar, cuya pantalla de acceso muestra la Figura 3, se estructura en torno a ocho módulos. Al principio, durante las dos primeras ediciones del Webinar, los siete primeros se centraban en el manejo general de la hoja de cálculo y el último se enfocaba en la aplicación de la utilización de la hoja de cálculo para la resolución de problemas de gestión. Sin embargo, los estudiantes mostraban especial interés en conocer otras utilidades de la hoja de cálculo, especialmente en el campo de las finanzas. Por ello, la edición celebrada en el curso 2006/2007, incluyó, además del módulo de resolución de problemas de costes, dos dedicados a funciones financieras y a herramientas de análisis de sensibilidad, respectivamente. El último Webinar impartido continúa estructurándose en ocho secciones, pero sólo cinco de ellas se refieren al funcionamiento general de la hoja de cálculo (algunas lecciones y tareas se han agrupado 
para reducir el número total de módulos, pero los contenidos son mayores que en las ediciones anteriores).

Figura 3. Pantalla de acceso al "Tercer Webinar: La hoja electrónica de cálculo como herramienta de apoyo a la elaboración de información de gestión"

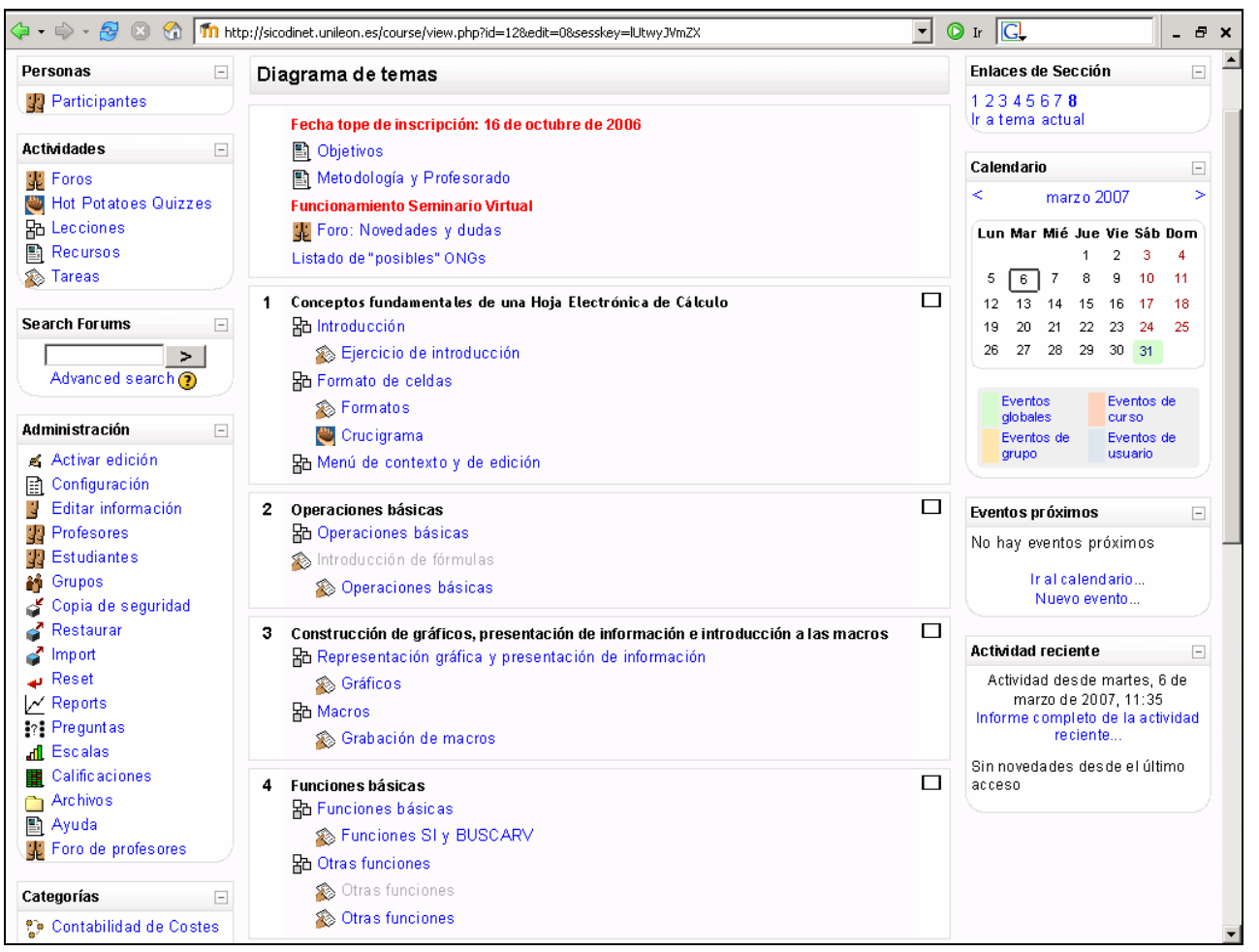

Los bloques con que cuenta el Webinar son los siguientes:

- Conceptos fundamentales de una hoja electrónica de cálculo. El objetivo es que los alumnos tengan un primer acercamiento con la hoja de cálculo: cómo se estructura, cómo introducir datos, cómo dar formato a las celdas, etc.

- Operaciones básicas. Su intención es conocer la introducción de fórmulas y funciones, la utilización de las referencias absolutas, los métodos para copiar y pegar, etc.

- Construcción de gráficos, presentación de información e introducción a las macros. El propósito es aprender a crear gráficos que representen la información introducida en una hoja de cálculo, que el alumno aprenda a seleccionar la información que desea de una hoja de 
cálculo, bien sea para imprimirla o para trasladarla a otro programa $y$, por último, pretende que los alumnos aprendan qué es y cómo diseñar una macro.

- Funciones básicas. Se describen, de forma general, las funciones que tiene Excel y se detallan algunas de ellas.

- Funciones financieras. Esta nueva sección tiene por objetivo conocer y aplicar las funciones de carácter financiero de uso general.

- Herramientas para el análisis de sensibilidad "Qué pasaría si...". Se muestran diferentes herramientas de la hoja de cálculo, como Solver, Escenarios o Tablas, que permiten crear opciones para facilitar la toma de decisiones o lograr un objetivo determinado.

- Elementos estéticos de la hoja de cálculo. Es el único módulo voluntario del seminario y pretende que los alumnos aprendan a mejorar la apariencia de una hoja de cálculo con la introducción de botones de control.

- Ejercicios de recapitulación. Su objetivo es aplicar los conocimientos adquiridos en los módulos anteriores a problemas típicos de Contabilidad de Costes.

\subsection{Análisis de la operativa del aprendizaje}

El desarrollo de esta actividad se ha planteado utilizando los diferentes recursos disponibles en Moodle, si bien el alumnado no suele tener experiencia en actividades on-line, por lo que previamente se pueden consultar las explicaciones sobre las pautas generales de funcionamiento. A través de unas sencillas presentaciones se muestra la articulación del Webinar en la plataforma Moodle, explicando los diferentes paneles que proporcionan acceso a los tópicos y recursos del mismo (http://sicodinet.unileon.es/mod/resource/DOCEN/Seminario/Funcionami ento_seminario.pps).

En lo referente al desarrollo del propio seminario, los alumnos acceden de forma secuencial a los contenidos teóricos a través de la web, en los recursos denominados "Lecciones" de Moodle (Figura 4). Una vez que los alumnos superan los contenidos teóricos de cada lección se les permite el acceso a las tareas o ejercicios correspondientes a dichos contenidos (Figura 5), solicitándoles la realización de las mismas. Cuando envían sus tareas, éstas van siendo evaluadas por los profesores, los cuales proceden a establecer las oportunas calificaciones $\mathrm{y} / \mathrm{o}$ correcciones 
que se consideran necesarias. El alumno puede rectificar aquellos errores detectados por los profesores y reenviar sus trabajos. Finalmente, a medida que dichas tareas son evaluadas positivamente, el alumno irá avanzando en los contenidos hasta completar el programa del Webinar.

Figura 4. Sicodinet. Webinar: Ejemplo de lección

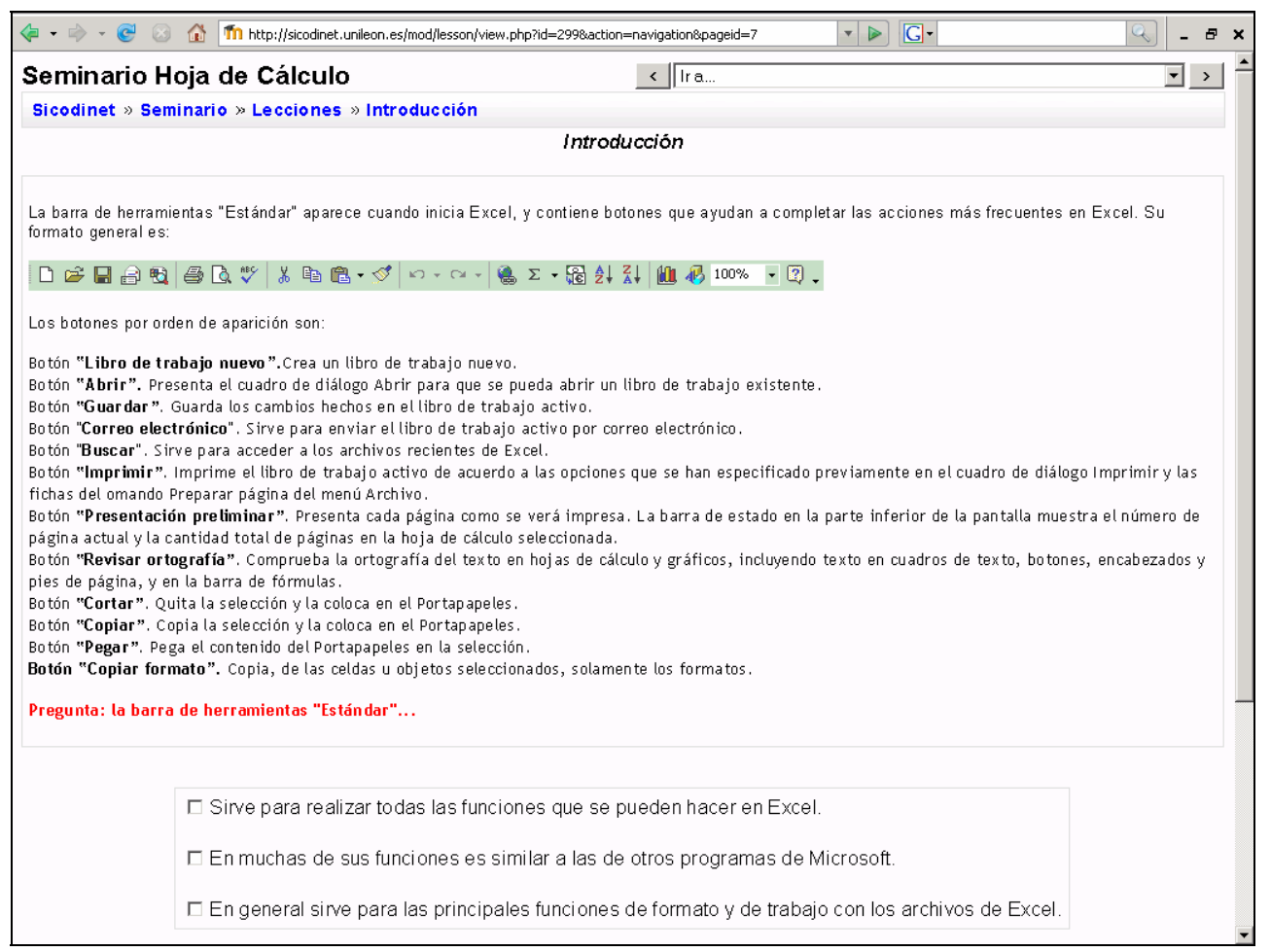

La principal ventaja en este tipo de actividades es la posibilidad de intercambiar dudas y soluciones a las mismas a través del foro del Webinar. Así, las dudas surgidas durante el estudio individual son planteadas por los alumnos en el foro, pudiendo ser contestadas por cualquiera de los participantes, por lo que no sólo se avanza en el conocimiento de forma autónoma sino que los alumnos contribuyen al aprendizaje del resto de compañeros, lo que también les sirve para comprobar, su propio nivel de conocimiento.

Respecto a la duración, el Webinar comienza a principios de octubre y termina a finales de diciembre, por lo que los estudiantes cuentan con más de dos meses para acceder a las lecciones y realizar las 
tareas prácticas. Además, existe un calendario que muestra la fecha límite para el envío de tareas; generalmente los alumnos tienen una semana para hacer dos tareas, pero es un tiempo flexible porque todos los módulos son accesibles desde el inicio. Así, aquellos que lo deseen pueden avanzar y enviar tareas programadas para semanas posteriores. Este hecho facilita que cada alumno adapte el progreso en el Webinar a su propio proceso de aprendizaje.

Figura 5. Sicodinet. Webinar: Ejemplo de tarea

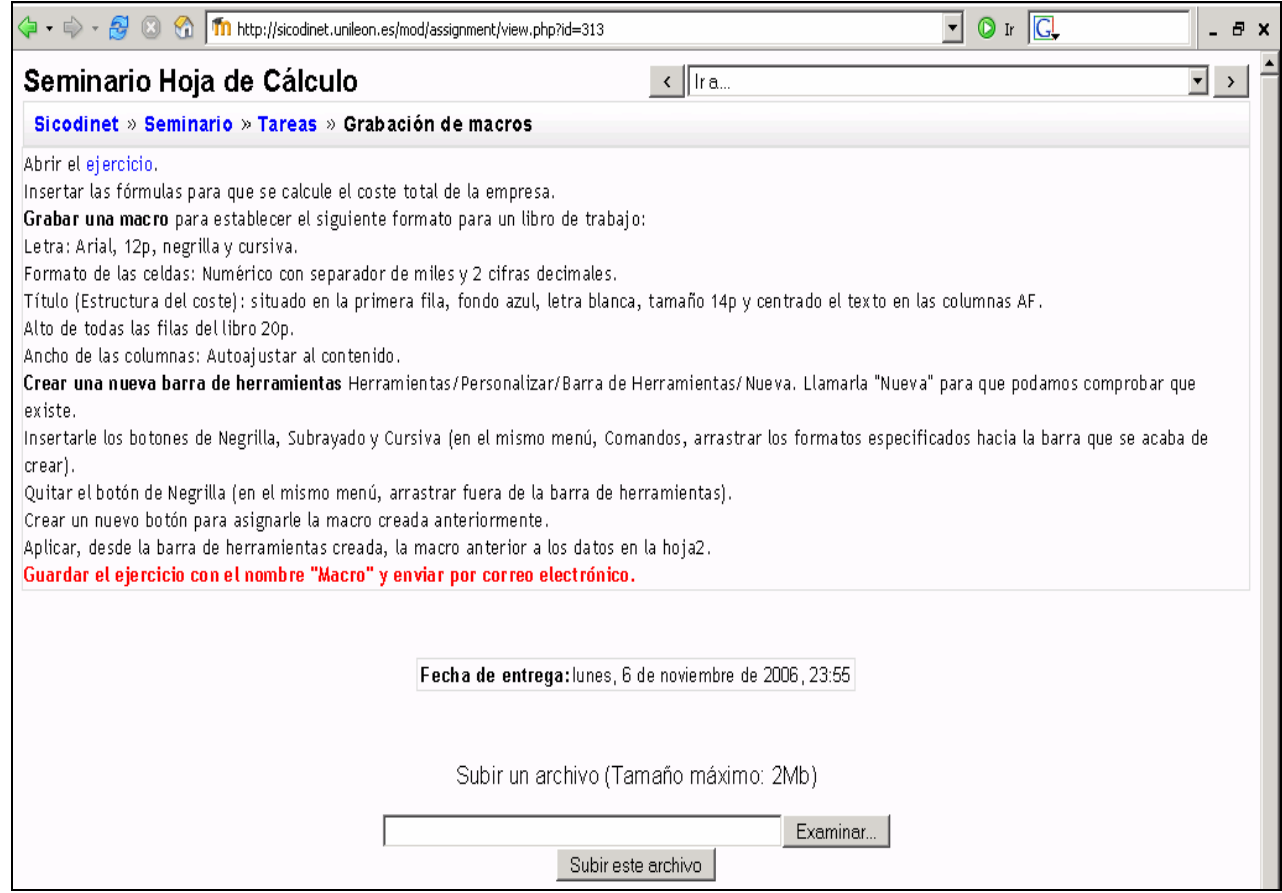

\subsection{Análisis de resultados}

En relación a los alumnos participantes en esta iniciativa, cabe señalar que el número de alumnos que optó por la versión on-line fue superior a la versión presencial en el año 2004/2005. En ese curso, el $80 \%$ de los que iniciaron el mismo, lo concluyeron con éxito, mientras que en las siguientes ediciones este porcentaje se ha reducido: en el curso $2005 / 2006$ terminaron el Webinar el $68 \%$ de los inscritos y en el $2006 / 2007$ solamente el $48 \%$. 
Además, desde curso académico 2005/2006, al terminar todas las tareas, se solicita a los alumnos que completen vía Internet y reenvíen un cuestionario anónimo acerca Webinar (http://sicodinet. unileon.es/encuesta/index.php?sid=3). Éste se estructura en torno a cuatro aspectos: funcionamiento, metodología, contenidos y otras consideraciones, siendo 47 el total de cuestiones. A continuación se detallan algunas de las respuestas dadas por los participantes.

En cuanto al tiempo de duración del seminario, la Figura 6 muestra las respuestas dadas por los alumnos; la mayoría estima que la duración del seminario es normal $(76,74 \%$ y $63,23 \%$, en los cursos $2005 / 2006$ y 2006/2007, respectivamente), aunque en ambos cursos académicos un porcentaje significativo opina que debería alargarse un poco. Los alumnos que opinan que debería reducirse su duración son más en el último curso $(11,54 \%)$ de celebración del Webinar que en el 2005/2006 (2,33\%).

Figura 6. Valoración de los alumnos sobre la duración del Webinar

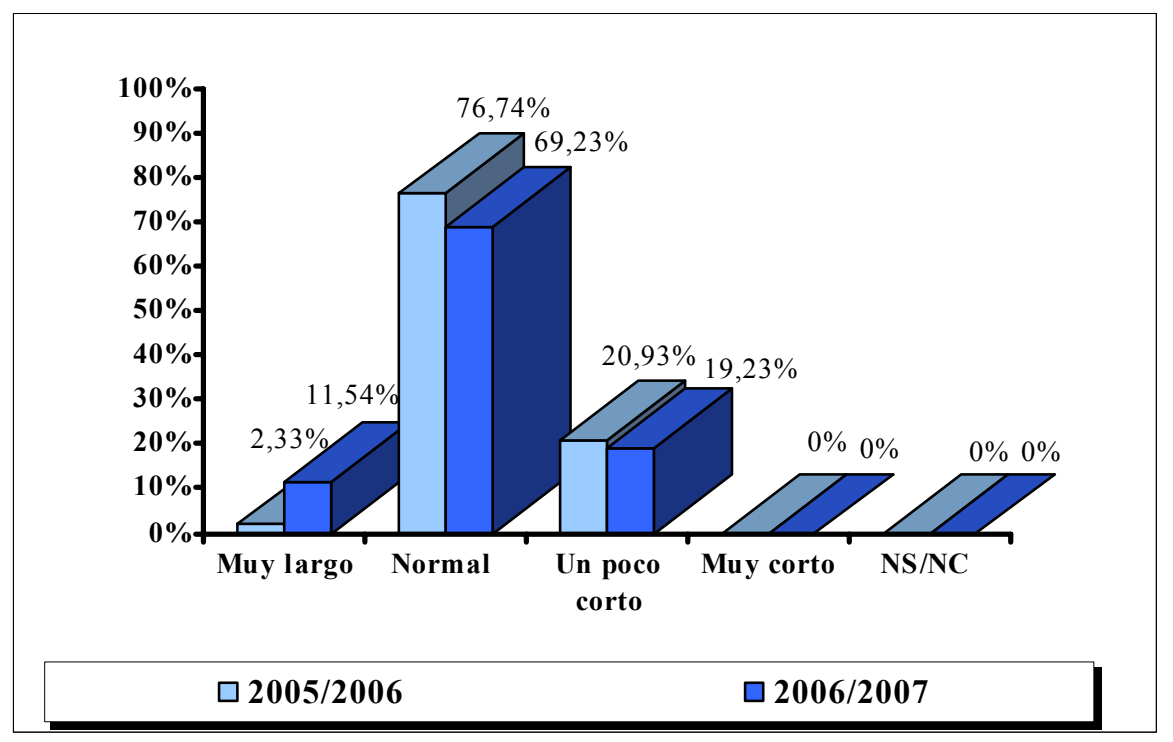

En relación a las tareas realizadas en el Webinar, de nuevo la gran mayoría las califican como "asequibles", como se expone en la Figura 7. Muy pocos consideran que las tareas son "complicadas" $(11,63 \%$ en $2005 / 2006$ y $11,54 \%$ en 2006/2007). Sólo en el curso 2005/2006 un $4,65 \%$ de los alumnos que realizaron el cuestionario consideraban que las tareas eran "fáciles". 
Figura 7. Valoración de los alumnos sobre la dificultad de las tareas del Webinar

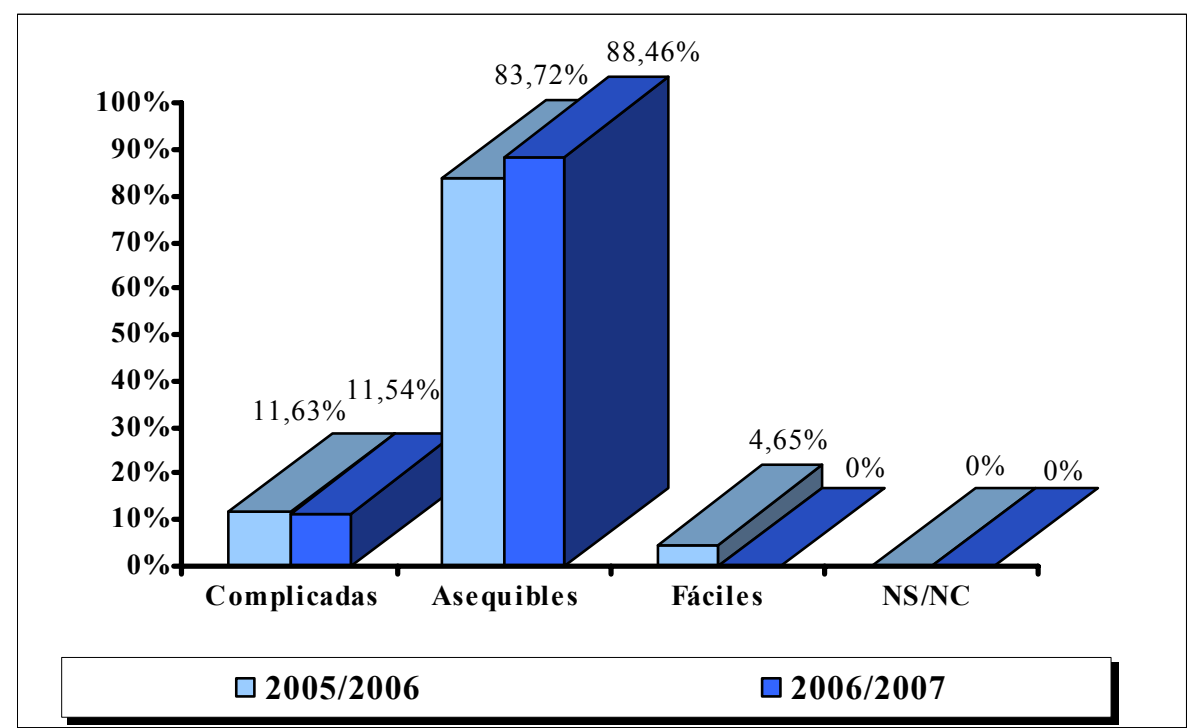

Respecto a la existencia de un foro para la resolución de dudas, en los dos cursos académicos en que se realizó el cuestionario, todos los alumnos coincidieron en señalar, según se muestra en la Figura 8 , que es "muy útil" o que "puede resultar de utilidad", aunque la gran mayoría coincide en la valoración más óptima.

Figura 8. Valoración de los alumnos sobre la utilidad del foro en el Webinar

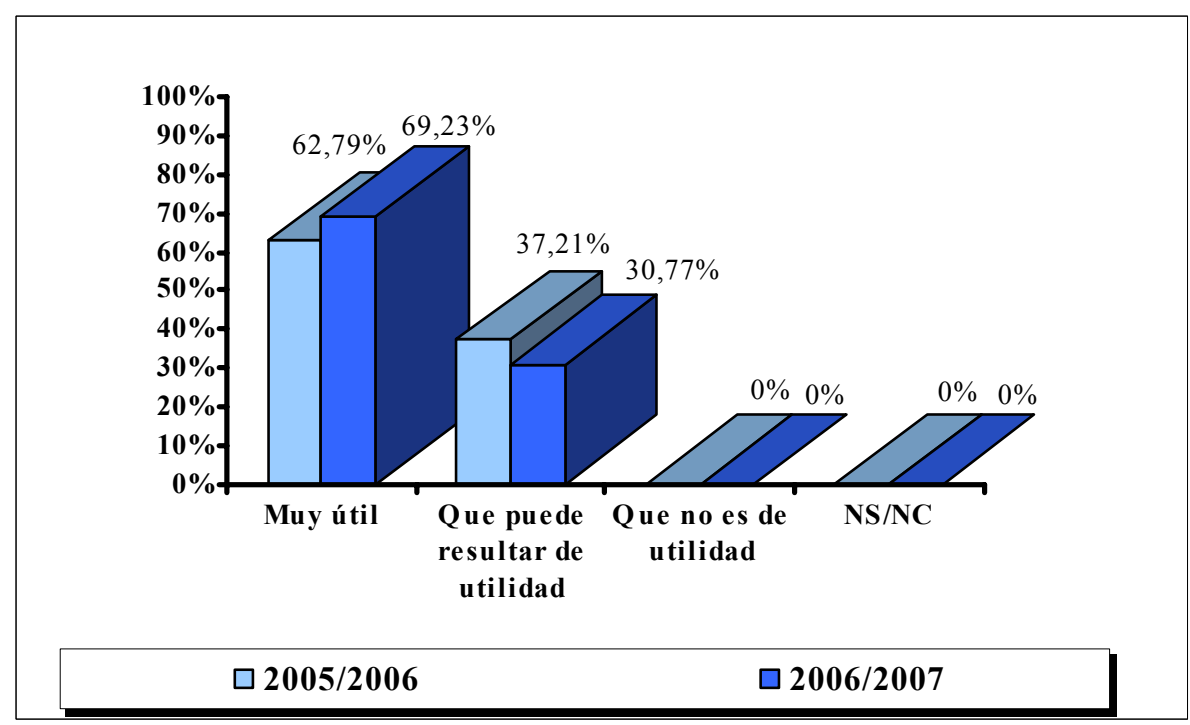


La participación de los alumnos en el foro se valora con diferente distribución en las dos ediciones del Webinar, según se expone en la Figura 9: en 2005/2006 las opiniones se reparten con porcentajes similares valorando la intervención como "normal" (48,86\%) o "escasa" (43,51\%), mientras que en el curso 2006/2007 el porcentaje que la considera "normal" $(65,38 \%)$ es bastante superior al que la consideran "escasa" (26,92\%).

Figura 9. Valoración de los alumnos sobre la participación en el foro del Webinar

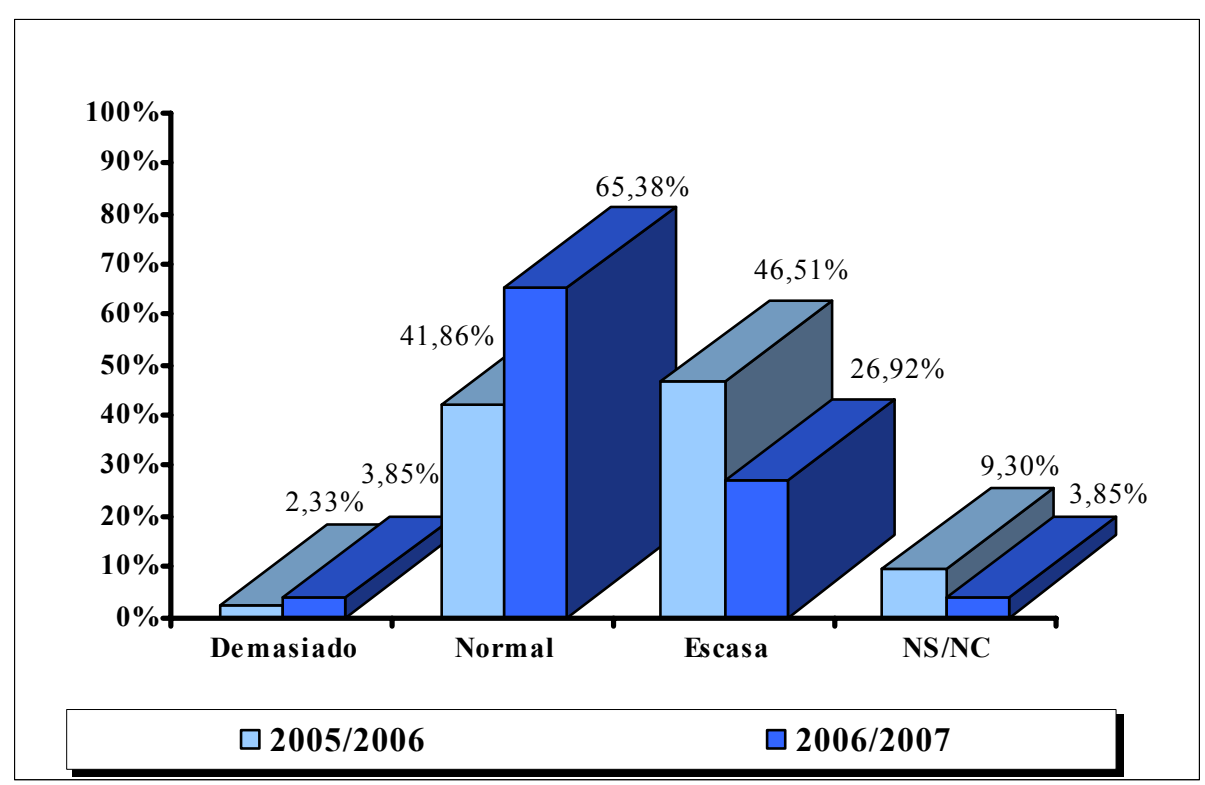

Por último, según se refleja en la Figura 10, la gran mayoría considera que la ayuda prestada por los profesores para el desarrollo de las tareas ha sido "suficiente" $(97,67 \%$ en $2005 / 2006$ y $88,46 \%$ en 2006/2007), aunque en la última edición son más los que opinan que ha sido "escasa" $(11,54 \%)$ frente al 2,33\% del curso 2006/2007.

En general, las opiniones obtenidas por parte de los alumnos participantes son muy positivas, lo que nos anima continuar con su celebración en los próximos cursos académicos. 
Figura 10. Valoración de los alumnos sobre la ayuda prestada por los profesores en el Webinar

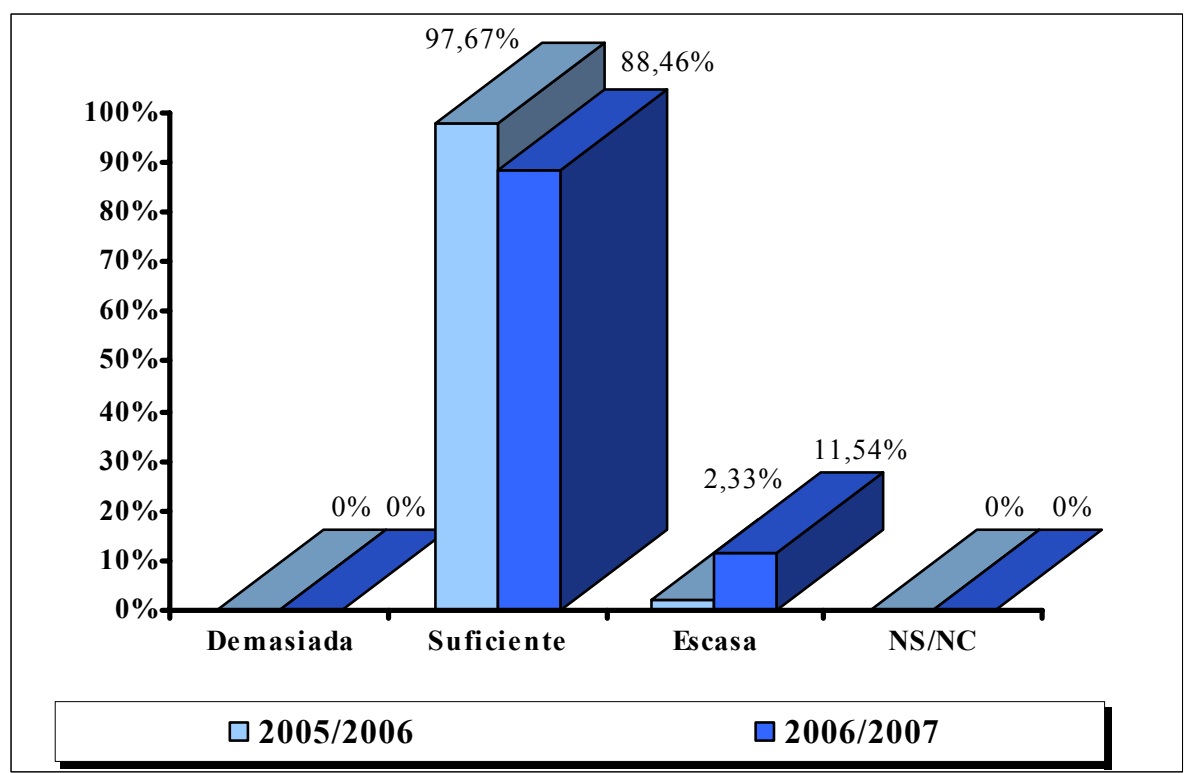

\subsection{Lecciones aprendidas}

Después de las ediciones celebradas del Webinar sobre hoja de cálculo, las conclusiones obtenidas al respecto son varias y, aunque la satisfacción de los alumnos y los profesores es bastante generalizada, también existen inconvenientes y riesgos en torno a esta experiencia.

En cuanto a las limitaciones, se puede destacar la escasa planificación realizada por muchos de los alumnos del seminario; al tratarse de un aprendizaje no presencial, en el que los participantes dedican el tiempo que estimen necesario para adquirir nuevos conocimientos y realizar las tareas, en ocasiones los mismos no establecen una dedicación mínima para avanzar en el seminario, pensando que cuentan con tiempo suficiente, pero su progreso se va ralentizando y terminan por desmotivarse. Ésta puede constituir una de las causas que explique la disminución progresiva del número de alumnos que finaliza el seminario durante las tres ediciones de celebración. Como consecuencia de lo anterior, se desprende que se corre el riesgo de considerar que este tipo de iniciativas requieren menor esfuerzo y trabajo, cuando el hecho es bien distinto, ya que los alumnos deben realizar solos una primera aproximación a los contenidos, lo que, obviamente, implica un esfuerzo adicional. 
Otro inconveniente surge con la experiencia previa de los alumnos en el manejo de la hoja de cálculo, lo que provoca que existan grupos con diferentes niveles de conocimiento. Por ello, el ritmo de trabajo, sobre todo al inicio del Webinar, resulta bastante desacompasado ya que los primeros módulos son bastante sencillos para aquellos que ya hayan tenido una aproximación a la hoja de cálculo.

Asimismo, los alumnos suelen tener miedo a la hora de utilizar el foro como escenario para plantear sus dudas y en muchas ocasiones no responden a las mismas, siendo el profesor el interlocutor casi absoluto de sus respuestas. Esta situación conlleva a que los participantes desperdicien un medio de aprendizaje que puede plantearles una mayor riqueza de aprendizaje, al facilitar ayuda a otros y establecer un intercambio de opiniones ante un problema. El foro, como cualquier medio de participación colectiva, puede constituirse como un escenario óptimo para construir conocimiento, así como el contexto ideal para fomentar la motivación de los alumnos.

A pesar de los inconvenientes señalados, la experiencia también ha reportado consecuencias positivas $y$ ha propiciado el planteamiento de nuevas oportunidades de trabajo. Así, los alumnos han adaptado su progreso en los contenidos del seminario según su propio proceso de aprendizaje, lo que les ha facilitado aprender a su propio ritmo. En consecuencia, los participantes más aventajados progresan sin necesidad de esperar por el resto de compañeros, al tiempo que pueden ayudar a los más rezagados a resolver dudas. Asimismo, este tipo de iniciativas permite la creación de grupos de trabajo homogéneos, compuestos por alumnos con un mismo nivel de conocimiento sobre la hoja de cálculo, o interdisciplinares, formados por alumnos con diferentes habilidades o grados de conocimiento, que abarquen diferentes contenidos del seminario, avanzando de forma conjunta o sustentando el aprendizaje del resto. Estos grupos de trabajo permitirían profundizar en los módulos del seminario y también plantear temas de debate más detallados en el foro del Webinar.

Además del foro, también se plantea la posibilidad de establecer, según señalan varios alumnos en el cuestionario, una sesión semanal de chat para reunir de forma sincrónica a los participantes. Esta opción puede ayudar a combatir la timidez de los alumnos a la hora de participar en el foro ya que se trata de un medio que suelen utilizar en otros contextos y, por tanto, con el que están más familiarizados. 
Por otro lado, una posible solución para evitar la desmotivación de los alumnos consistiría en la celebración de al menos dos sesiones presenciales que sirvan para exponer las directrices generales del Webinar, así como aclarar aquellos aspectos de la hoja de cálculo que puedan resultar de mayor dificultad.

\section{CONCLUSIONES Y LÍNEAS FUTURAS}

La creación del Espacio Europeo de Educación Superior (EEES) implica la realización de numerosos cambios, especialmente en la formulación del proceso de enseñanza-aprendizaje, ya que el alumno pasa a ser el eje central del proceso. Desde esta perspectiva, surge el enfoque basado en "aprender a aprender" en el que el alumno es el gestor de su propio proceso educativo y el profesor actúa como guía del mismo.

En el EEES se diferencia entre horas presenciales y horas no presenciales de los alumnos, dividiéndose estas últimas en trabajo personal del alumno (realización de trabajos individuales o en grupo, búsqueda de bibliografía e información, estudio individual para la preparación de exámenes, prácticas sin la presencia del profesor, prácticas en empresas) y horas de contacto directo con el profesor (seminarios, realización de exámenes, tutorías del profesor para la realización de trabajo y otras tutorías individualizadas). Además, se pretende que los alumnos se familiaricen con diferentes herramientas informáticas, con independencia de la asignatura concreta en que se enmarque la docencia.

Precisamente, el Webinar presentado en este trabajo permite alcanzar los objetivos señalados: establece mecanismos que guían al alumno en la construcción de su propio conocimiento y promueve la utilización de de herramientas informáticas.

El desarrollo del seminario en línea ha supuesto desde el punto de vista del profesorado la necesidad de adaptarse a nuevas herramientas metodológicas, como el manejo de la plataforma que ha soportado el funcionamiento del seminario (Moodle), el diseño de los contenidos o la implementación en la Web de lecciones, tareas, ejercicios, etc.

Asimismo, ha sido necesario un esfuerzo adicional en tiempo de respuesta a los participantes en el seminario, ya que la metodología 
que se ha seguido implica no sólo la respuesta a las dudas de los alumnos sino también la corrección de las tareas que ellos realizan, lo que conlleva dedicar bastante tiempo a esta labor, ya que si las tareas no están resueltas correctamente los alumnos deben corregir los errores y reenviarlas de nuevo, con lo que muchas veces el trabajo de revisión y corrección de los profesores del Webinar se duplica.

A pesar del mayor esfuerzo que la actividad citada conlleva, se pretende continuar celebrando el seminario virtual en los cursos académicos futuros y, para mejorar la calidad y los contenidos del mismo, se plantean los siguientes propósitos al respecto:

- Incluir un apartado que se recoja las respuestas a dudas y preguntas frecuentes sobre el Webinar, con la finalidad de que los futuros participantes puedan consultarlas sin la necesidad de plantearlas en el foro.

- Desarrollar un seminario más avanzado sobre la aplicación de hoja de cálculo a Contabilidad de Costes. La celebración de las diferentes ediciones del seminario virtual ha tenido lugar en el primer trimestre del curso académico, por lo que el temario de la asignatura de Contabilidad de Costes no se ha estudiado en su totalidad. Por ello, en futuros cursos se pretende ampliar el seminario con tareas que estén relacionadas con la totalidad del temario de la asignatura. Esto también permitirá que los participantes perfeccionen el uso de hoja de cálculo y comprendan la utilidad de esta herramienta para el análisis y la resolución de problemas y para la toma de decisiones.

\section{BIBLIOGRAFÍA}

ACCINO, J.A. (2002) "Un entorno de componentes para enseñanza virtual basada en software libre", Novatica, 156. Disponible en: http://www. ieev.uma.es/ieev/filos.htm (7 de marzo de 2007).

Accounting Education ChANGe COMmission (AECC) (1990) "Objectives of Education for Accountants", Position Statement, 1, septiembre.

AdELL, J. (1997) "Tendencias en educación en la sociedad de las tecnologías de la información", Edutec, Revista Electrónica de Tecnología Educativa, 7, noviembre. Disponible en: http://www.uib.es/depart/gte/edutece/revelec7/revelec7.html (7 de marzo de 2007). 
Alonso, C. y Gallego, D. (1996) "Formación del profesor en Tecnología Educativa". D. GALlEGO, C. ALONSO y I. CANTÓN (coords.) Integración curricular de los recursos tecnológicos. Barcelona: Oikos-Tau, pp. 3164.

BARONE, C.A. y LUKER, M.A. (1999) "The Role of Advanced Networks in the Education of the Future", Educom Review, noviembre/diciembre. Disponible en: http://www.educause.edu/ir/library/html/erm/erm99/ erm9968.html (7 de marzo de 2007).

BARTOlOmÉ, A. (2004) "Blended Learning. Conceptos básicos", Revista Píxel-Bit, Revista de Medios y Educación, Monográfico Blended Learning, 23, pp. 7-20.

CABERO, J. (1995) "Nuevas tecnologías, comunicación y educación", EDUTEC, Revista Electrónica de Tecnología Educativa, 1. Disponible en: http://www.sav.us.es/pixelbit/articulos/n23/n23art/art2301.htm (7 de marzo de 2007).

CHALMERS, J. (1998) "Virtual Education". Disponible en: http://underground. musenet.org:8080/bkort/EdMud.html (7 de marzo de 2007).

CoLL, C. (1994) "El análisis de la práctica educativa: reflexiones y propuestas en torno a una aproximación multidisciplinar", Tecnología y Comunicación Educativas, 24, pp. 3-29.

CRUE (2002) "Declaración de Bolonia: Adaptación del sistema universitario español a sus directrices", 14 de diciembre.

- (2002) "La Declaración de Bolonia y su repercusión en la estructura de las titulaciones en España. Acuerdo de la Asamblea General de la CRUE", 8 de julio.

- (2003) "Declaración de la Conferencia de Rectores de las Universidades Españolas sobre el Espacio Europeo de Educación Superior", 6 de octubre.

- (2003) "Declaración de la Conferencia de Rectores de las Universidades Españolas sobre el Espacio Europeo de Enseñanza Superior", 12 de septiembre.

De Pablos Pons, J. (1999) "Las tecnologías de la información y la comunicación: un punto de vista educativo", Cuadernos de Documentación Multimedia, 8. Disponible en: http://www.quaderns digitals.net/index.php?accionMenu=heme-roteca.VisualizaArticulolU.vi sualiza\&articulo_id=3033 (7 de marzo de 2007).

DI ANTONIO, A. (1987) Las hojas electrónicas y su aplicación en la empresa. Bilbao: Deusto. 
FERNÁNDEZ MUÑOZ, R. (2001) "El profesor en la sociedad de la información y la comunicación: nuevas necesidades en la formación de profesorado", Revista Digital Docencia e Investigación, 1. Disponible en: http://www. uclm.es/-profe-sorado/ricardo/Docencia_e_Investigacion/Docencia_e_ Investigacion.htm (7 de marzo de 2007)

IMBERNÓN MUÑOZ, F. (2001) "La profesión docente ante los desafíos del presente y del futuro". C. MARCELO (coord.) La función docente. Madrid: Síntesis.

IZARD, C. y ReEVE, J. (1986) "Electronic Spreadsheet in the teaching of Accounting and Taxation. Uses, limitations and examples", Journal of Accounting Education, vol. 4, 1, pp. 161-175.

LARA, P. y DUART, J.M. (2005) "Gestión de contenidos en el e-learning: acceso y uso de objetos de información como recurso estratégico", Revista de Universidad y Sociedad del Conocimiento, vol. 2, 2, noviembre. Disponible en: http://www.uoc.edu/rusc/2/2/dt/esp/lara. pdf (7 de marzo de 2007).

LÓPEZ GONZÁLEZ, E. (1991) "Tecnologías de la información y enseñanza universitaria de la Contabilidad en España", IV Actas del Encuentro de Profesores Universitarios de Contabilidad, Santander.

- y MENDAÑA Cuervo, C. (2005) "Espacio Virtual de Aprendizaje SICODINET: Hacia una Formación para la Autonomía". Primer Premio a la Innovación a la Enseñanza 2005, Consejo Social, Universidad de León. Disponible en http://sicodinet.unileon.es/dir/docs/sicodinetinnovacio neducativa.pdf (7 de marzo de 2007).

-; - y GonZÁlez PÉREZ, B. (2006) "La experiencia del Seminario Virtual: La hoja electrónica de cálculo como herramienta de apoyo a la elaboración de información de gestión en el Espacio Virtual de Aprendizaje SICODINET". Actas del VI Congreso Internacional Virtual de Educación (CIVE'06). CD-Rom (ISBN 84-7632-978-4).

-; - y - (2006) "It's a Bird... It's a Plane... It's SICODINET: a B-learning Experience in Management Accounting in University of León". Proceedings of the 8th International Symposium on Computers in Education (SIIE'06). León, vol.1, pp. 408-416.

Mendaña Cuervo, C. y González Pérez, B. (2004) "El papel de las WebQuest como herramienta para el aprendizaje del alumno en la nueva sociedad del conocimiento". Comunicación presentada en el III Simposio Virtual de Computación en la Educación (SOMECE 2004). 
-; LóPez González, E. y González Pérez, B. (2005) "El caso del Espacio Virtual de Aprendizaje SICODINET". Actas del Congreso Hacia donde va la educación europea y americana. León, pp. 229-236.

MichaVILA PITARCH, F. (2001) "¿Soplan vientos de cambios universitarios?", Boletín de la Red Estatal de Docencia Universitaria, Vol. 1, 1, pp. 9-12.

- (investigador principal) (2003) "Potenciación de la acción tutorial basada en las Tecnologías de la Información", Laboratorio de investigación en Tecnologías de la Información y Cátedra UNESCO de Gestión Política Universitaria (http://liti.dmami.upm.es/tutorias).

MONEREO, C. y POZO, J.I. (2003) La universidad ante la nueva cultura educativa. Madrid: Síntesis.

MORA, J.G. y VIDAL, J. (2000) "Cambios en la educación superior en España: avances y nuevos conflictos", Revista de Educación Superior, 114, abril-junio, pp. 111-122.

MORENo, F. y BAULlY-BAILLIÈRE, M. (2002) Diseño instructivo de la formación on-line. Aproximación metodológica a la elaboración de contenido. Barcelona: Ariel.

PéreZ, R.; LóPeZ, A.J.; Fernández, A.M. y SOMARRIBA, N. (1999) "Teleenseñanza en la Universidad. El Proyecto AulaNet", Boletín RedIRIS, 5051, pp. 13-16.

REAL DECRETO 1497/1987, de 27 de noviembre, por el que se establecen directrices generales comunes de los planes de estudio de los títulos universitarios de carácter oficial y validez en todo el territorio nacional.

ReAL DeCRETO 1125/2003, de 5 de septiembre, por el que se establece el sistema europeo de créditos y el sistema de calificaciones en las titulaciones universitarias de carácter oficial y validez en todo el territorio nacional.

ReAl DeCReto 55/2005, de 21 de enero, por el que se establece la estructura de las enseñanzas universitarias y se regulan los estudios universitarios oficiales de Grado.

RINAUDO, M.C. y VÉLEZ, G. (2000) Estrategias de Aprendizaje y Enfoque Cooperativo. Córdoba: Educando Ediciones.

SALINAS, J. (1994) "Educación a distancia basada en satélites: experiencias y perspectivas". F. BlazQueZ, J. CABero y F. LosCerTales (coord.) Nuevas Tecnologías de la Información y Comunicación para la Educación. En Memoria de J. Manuel López Arenas. Sevilla: Alfar, pp. 55-64. 
- y SUREDA, J. (1992) "Aprendizaje abierto y educación a distancia". J.M. SANCHO (coord.) European Conference about Information Technology in Education: A Critical Insight. Proceedings Congrés Europeu T.I.E. Barcelona, pp. 677-686.

SMITH-GRATto, K. (2000) Instructional and Cognitive Impacts of Web-Based Education. Londres: Idea Group Publishing.

Tua Pereda, J. (1995) "En torno a la enseñanza de la Contabilidad. Una reflexión personal", Técnica Contable, 557, pp. 313-334 y 350.

UE (1998) "Joint declaration on harmonisation of the architecture of the European higher education system (Declaración de La Sorbona)", Consejo de Ministros de Educación Superior, París, 25 de mayo.

UE (1999) "Declaración de Bolonia", Consejo de Ministros de Educación Superior, Bolonia, 19 de junio.

UE (2001) "Comunicado de Praga", Consejo de Ministros de Educación Superior, Praga, 19 de mayo.

UE (2003) "Realising the European Higher Education Area. Comunicado de Berlín", Consejo de Ministros de Educación Superior, Berlín, 18-19 septiembre.

VALCÁRCEL CASES, M. (coord.) (2003) "La preparación del profesorado universitario español para la convergencia europea en educación superior". Proyecto del Ministerio de Educación y Ciencia (EA2003-004). Disponible en: http://wwwn.mec.es/univ/html/informes/estudios_ana lisis/resultados_2003/EA2003_0040/informe_final.pdf (7 de marzo de 2007). 